\title{
Communications
}

Schweizerische Vereinigung
psychiatrischer Chefärzte

\section{Integrierte Versorgung}

statt Drehtürpsychiatrie

Die Zahl der stationären psychiatrischen Behandlungen hat im Zeitraum von 2000 bis 2006 um 9,3\% zugenommen, wie eine Studie des Schweizerischen Gesundheitsobservatoriums ergab [1]. Brisant an dieser Entwicklung ist, dass die Anzahl der behandelten Personen nicht zugenommen hat. Vielmehr erklärt sich die Zunahme der Fälle durch deutlich häufigere Wiedereintritte (plus 30\%) bei stetig abnehmender Aufenthaltsdauer.

Laut psychiatrischen Experten spricht das Ergebnis für einen häufigeren Wechsel zwischen ambulanten und stationären Behandlungen. Die Entwicklung in Richtung kürzerer, aber allenfalls häufigerer Klinikeintritte wird somit als grundsätzlich positiv für Patientinnen und Patienten beurteilt. Voraussetzung dafür ist aber eine gute Vernetzung ambulanter und stationärer Angebote im Sinne einer integrierten und durchlässigen Versorgung.

Die psychiatrischen Chefärztinnen und Chefärzte der Schweiz unterstützen die Entwicklung zu einer integrierten Versorgung und setzen sie in ihren Institutionen um. Sie weisen darauf hin, dass zuerst ambulante Behandlungsangebote aufgebaut werden müssen, bevor Betten in den Kliniken reduziert werden. Sonst besteht die bekannte Gefahr der Drehtürpsychiatrie: Patientinnen und Patienten werden aus Platzmangel $\mathrm{zu}$ früh entlassen, haben ungenügende ambulante Behandlungsmöglichkeiten und treten kurz darauf wieder in die psychiatrische Einrichtung ein. Kürzere Aufenthaltsdauer und häufigere Eintritte haben zu einer spürbaren Verdichtung der Arbeit in psychiatrischen Kliniken und zu einer stärkeren Belastung des Fachpersonals geführt. Die Attraktivität der Arbeitsplätze in psychiatrischen Institutionen muss angesichts des knappen Angebots von Fachpersonal gezielt gefördert werden. Dies in erster Linie durch genügende personelle Ressourcen zum Schutz vor Überlastung, aber auch durch weitere Massnahmen im Bereich der Weiterbildung und der Organisation.

1 Stationäre Psychiatrie in der Schweiz 2000-2006. obsan fact sheet. Neuenburg: Schweizerisches Gesundheitsobservatorium (Obsan); 2008.

Ärztinnen und Ärzte
für Umweltschutz

Zusammenhang zwischen Hochspannungsleitungen und Alzheimerrisiko

Mitten im geplanten Ausbau des schweizerischen Hochspannungsnetzes zeigt eine Studie der Berner Universität ein erhöhtes Alzheimerrisiko um Hochspannungsleitungen in der Schweiz. Dieses Studienergebnis ist ein weiterer gewichtiger Hinweis dafür, dass der Gesundheitsschutz durch die Grenzwerte der seit Februar 2000 geltenden NIS-Verordnung nicht ausreichend gewährleistet ist. Es besteht Handlungsbedarf, um dem im Umweltschutzgesetz verankerten Grundsatz der Vorsorge besser Rechnung zu tragen

\section{Fakten}

Wer mindestens 15 Jahre in der Nähe einer Hochspannungsleitung wohnt, zeigt aufgrund der neuen Untersuchung ein doppelt so hohes Risiko, an Alzheimer zu erkranken, im Vergleich zur restlichen Bevölkerung.

Alzheimer ist aber nicht das einzige Gesundheitsrisiko. Menschen, die in der Nähe von Hochspannungsleitungen wohnen, klagen aber auch über andere gesundheitliche Beeinträchtigungen wie Schlafstörungen, Kopfschmerzen oder Müdigkeit.

\section{Handlungsbedarf gegeben}

Die Ärztinnen und Ärzte für Umweltschutz unterstützen einen sinnvollen und effizienten Ausbau von Hochspannungsleitungen, sofern Umwelt- und Gesundheitsschutz gemäss dem aktuellen Wissensstand berücksichtigt werden. Es braucht Kriterien für die Reduktion der Magnetfelder bei Erdverlegung der Leitungen. Für die durch Hochspannungsleitungen verursachten Magnetfelder braucht es in bevölkerten Gebieten ein systematisches Monitoring, wobei den alten Hochspannungsleitungen ein besonderes Augenmerk gilt.

Verbindliche niedrigere Grenzwerte, die für alle Hochspannungsleitungen gelten, erachten wir als notwendig. Neben den Hochspannungsleitungen gilt es, Trafostationen in Schul- und Wohnhäusern abzuschirmen oder $\mathrm{zu}$ entfernen. Für Anwohnerinnen und Anwohner mit Beschwerden in Zusammenhang mit niederfrequenten Magnetfeldern fordern wir eine medizinische Abklärung unter Einbezug von Umweltspezialisten. Aus ärztlicher Sicht ist es sinnvoll, auch die hausgemachte Magnetfeldbelastung durch entsprechende Information und Aufklärung der Bevölkerung zu minimieren.

\section{Eidgenössische Kommission für Impffragen und Bundesamt für Gesundheit}

Beilage zu dieser Ausgabe: Factsheet «Schweizerischer Impfplan» (Stand September 2008)

Zurückgehend auf diverse Anfragen praktizierender Ärztinnen und Ärzte haben die Eidgenössische Kommission für Impffragen (EKIF) und das Bundesamt für Gesundheit ein Informationsblatt «Schweizerischer Impfplan» ausgearbeitet. Dabei handelt es sich um eine Zusammenfassung der Richtlinien und Empfehlungen «Schweizerischer Impfplan 2008» (www.sich-impfen.ch).

Dieses Factsheet ist in deutscher, französischer und italienischer Sprache erhältlich. Weitere Exemplare können unter der folgenden Adresse bestellt werden: Bundesamt für Bauten und Logistik (BBL), Vertrieb Publikationen, Postfach, 3003 Bern, Fax 03132550 58, E-Mail: verkauf.zivil@bbl.admin.ch, Internet: www.bundespublikationen.admin.ch, Bestellnummer: 311.267.d (f, i).

\section{Commission fédérale pour \\ les vaccinations et Office fédéral de la santé publique}

En annexe de ce numéro:

fact sheet «Plan de vaccination suisse» (état septembre 2008)

Afin de répondre à la demande d'un certain nombre de médecins praticiens, la Commission fédérale pour les vaccinations (CFV) et l'Office fédéral de la santé publique ont élaboré une fiche d'information «Plan de vaccination suisse» qui leur est destinée. Il s'agit d'un résumé du «Plan de vaccination suisse 2008» de la série Directives et Recommandations (www.sevacciner.ch).

Cette fiche existe en trois langues: français, allemand et italien. Des exemplaires supplémentaires peuvent être commandés à l'adresse suivante: OFCL, Distribution des publications fédérales, Case postale, 3003 Berne, fax 031 32550 58, e-mail: verkauf.zivil@bbl.admin.ch, Internet: www.bundespublikationen.admin.ch, $\mathrm{n}^{\circ}$ de commande: 311.267.f (d, i). 


\section{Deutsche Morbus Crohn /}

Colitis ulcerosa Vereinigung e.V. (DCCV)

\section{Forschungsförderung}

Primärsklerosierende Cholangitis (PSC)

Die Deutsche Morbus Crohn / Colitis ulcerosa Vereinigung e.V. (DCCV) schreibt eine Forschungsförderung mit 150000 Euro zur primärsklerosierenden Cholangitis (PSC) aus. Die Förderung wird durch eine private Spende ermöglicht.

Inhaltliche Ausrichtung: Ziel des Vorhabens ist die Förderung der Forschung zur primärsklerosierenden Cholangitis. Das geförderte Projekt soll Fragen der Pathogenese, Diagnostik oder Therapie der PSC thematisieren. Es sollen zum einen solche Forschungsvorhaben gefördert werden, die im Bereich der Grundlagenforschung anzusiedeln sind und von denen die Entwicklung neuer Ansätze zur Therapie der PSC zu erwarten sind. Darüber hinaus ist diese Forschungsförderung offen für Bewerberinnen und Bewerber mit innovativen Projektideen zur Diagnostik der PSC, (z. B. zur [Weiter-]Entwicklung nichtinvasiver diagnostischer Methoden), zur frühzeitigen Erkennung von Komplikationen der PSC (z. B. eines Cholangiokarzinoms) oder zur Therapie der PSC.

Bewerbungsfristen und Vergabeverfahren: Die Vergabe der Forschungsförderung erfolgt durch ein zweistufiges Antrags- und Begutachtungsverfahren. In der ersten Antragsstufe sind bis zum 31. März 2009 formlose englischsprachige Projektskizzen, deren Umfang 3-6 Seiten nicht überschreiten sollte, vorzulegen. Diese Antragsskizzen werden von einer unabhängigen und international besetzten Gutachterkommission bewertet. Mit dem Ergebnis der Begutachtung ist spätestens im Juni 2009 zu rechnen. In der zweiten Verfahrensstufe werden die Antragsteller der positiv bewerteten Projektskizzen unter Angabe eines Termins (3 Monate nach Bekanntgabe) zur Vorlage eines ausführlichen englischsprachigen Projektantrages (etwa 20 DIN-A4-Seiten) aufgefordert. Die vorgelegten förmlichen Förderanträge werden von einem international besetzten Gutachtergremium bewertet.

Einzelheiten über Vergaberichtlinien sind dem Leitfaden «DCCV-Forschungsförderung PSC» $\mathrm{zu}$ entnehmen, der ebenso wie weitere Ausschreibungen in der DCCV-Bundesgeschäftsstelle angefordert oder im Internet abgerufen werden kann: www.dccv.de/stipendien.

Zielgruppe und allgemeine Voraussetzungen: Die Forschungsförderung ist für in Deutschland, im deutschsprachigen Ausland und in den Benelux-Staaten tätige Wissenschaftlerinnen und Wissenschaftler vorge- sehen, die Forschungsexpertise auf dem Gebiet der PSC nachweisen können.

Kontakt: Folgende Korrespondenzadresse gilt für alle Ausschreibungen: DCCV e.V., Reinhardtstr. 18, D-10117 Berlin, Tel. +49 30200039250 (Christine Witte), Fax +49 30 2000392 87, E-Mail: cwitte@dccv.de, Internet: www.dccv.de.

Schweizerische Morbus Crohn /
Colitis ulcerosa Vereinigung

Forschungsförderungspreis zum Thema «Ernährung und chronisch entzündliche Darmerkrankungen»

Die Schweizerische Morbus Crohn / Colitis ulcerosa Vereinigung (SMCCV) verleiht zum zweiten Mal einen Forschungsförderungspreis auf dem Gebiet der beiden Erkrankungen Morbus Crohn und Colitis ulcerosa. Der mit 10000 Franken dotierte Preis wird einzig durch Spenden der Betroffenen und ihrer Angehörigen finanziert.

Inhalt: Der Forschungsförderungspreis soll als Inhalt das Thema «Ernährung und chronisch entzündliche Darmerkrankungen» haben. Insbesondere soll thematisiert werden, ob bestimmte Nahrungsmittel einen Einfluss auf die Erkrankungen haben, ob mit einer bestimmten Diät ein Schub verhindert werden kann, ob Medikamente einen Einfluss auf die Ernährung haben usw.

Termine: Arbeiten können bis zum 31. Dezember 2009 eingereicht werden. Die Verleihung des Preises findet im Rahmen der Generalversammlung der SMCCV im Frühjahr 2010 statt.

Allgemeine Voraussetzungen: Die Mitglieder der SMCCV haben an der Generalversammlung im Jahr 2005 beschlossen, sich näher mit dem Thema der Forschungsförderung zu befassen und im Interesse der CED-Patienten auf dem Wissenschaftsmarkt mitzubieten, wenn auch nur mit geringen Mitteln. Bedenkt man, wie viele Jungakademiker heute wertvolle wissenschaftliche Arbeiten ganz umsonst erarbeiten, so ergibt sich für uns die Chance, hier Ressourcen in unsere Richtung zu lenken, die derzeit im akademischen Gedankenraum verpuffen. In diesem Sinne erhofft sich die SMCCV eine möglichst rege Teilnahme am Wettbewerb um den Forschungsförderungspreis und eine Menge neuer Erkenntnisse, mit denen entweder den Patienten direkt geholfen werden kann oder mit deren Hilfe politischer Druck ausgeübt werden kann, um unseren Interessen zum Durchbruch zu verhelfen. Der Teilnehmerkreis, die Teilnahmebedingungen, Einreichekriterien usw. können in den Bestimmungen der SMCCV-Forschungsförde- rung eingesehen werden. Diese können Sie bei uns bestellen, oder Sie können sie auf unserer Homepage abrufen (www.smccv.ch).

\section{Association Suisse de maladie}

de Crohn / Colite ulcéreuse

Prix encourageant la recherche au sujet de «l'alimentation et les maladies inflammatoires chroniques de l'intestin»

L'Association Suisse de maladie de Crohn / Colite ulcéreuse (ASMCC) attribue pour la deuxième fois un prix encourageant la recherche dans le domaine des deux maladies, celle de Crohn et la colite ulcéreuse. Le prix, doté de 10000 Francs, est uniquement financé grâce à des dons des malades et de leur entourage.

Contenu: Le prix encourageant la recherche doit aborder le sujet de «l'alimentation et les maladies inflammatoires chroniques de l'intestin». Le thème doit en particulier chercher à savoir si des aliments déterminés auraient une influence sur ces maladies, si grâce à un régime déterminé une poussée pourrait être bloquée, si des médicaments auraient un effet sur l'alimentation, etc.

Dates: Les travaux peuvent être déposés jusqu'au 31 décembre 2009. L'attribution du prix a lieu dans le cadre de l'Assemblée générale de l'ASMCC au printemps 2010.

Conditions générales: Lors de leur Assemblée générale de 2005, les membres de l'ASMCC ont décidé de mettre en place une structure permettant d'encourager la recherche et, dans l'intérêt des patients avec MICI, de participer à l'effort scientifique, même avec de petits moyens. Quand on pense à ces nombreux jeunes universitaires qui de nos jours proposent des travaux scientifiques de qualité pour rien, il s'agit pour nous de saisir notre chance en apportant des ressources qui correspondent à notre cause, alors qu'elles manquent actuellement au sein des académies. C'est pourquoi l'ASMCC compte sur une participation active à ce concours du prix encourageant la recherche et apportant de nombreuses nouvelles connaissances qui peuvent, soit en aidant directement les malades soit en exerçant une pression politique, contribuer à atteindre nos intérêts.

Les domaines concernés, conditions, critères, etc. peuvent être consultés dans le règlement de l'encouragement à la recherche de l'ASMCC. Vous pouvez nous les commander ou les consulter sur notre page Internet (www.smccv.ch). 